\title{
Grating based devices in polymer optical fibre
}

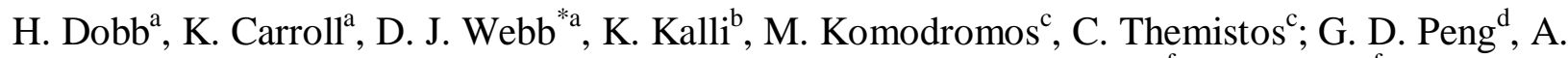

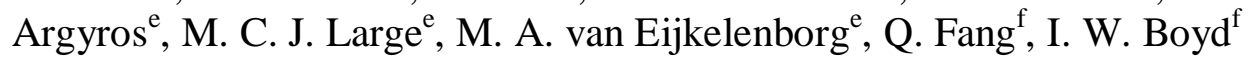 \\ ${ }^{\text {a } P h o t o n i c s ~ R e s e a r c h G r o u p, ~ A s t o n ~ U n i v e r s i t y, ~ A s t o n ~ T r i a n g l e, ~ B i r m i n g h a m, ~ B 4 ~ 7 E T, ~ U K ~}$ \\ ${ }^{\mathrm{b}}$ Higher Technical Institute, Cyprus \\ ${ }^{c}$ Frederick Institute of Technology, Cyprus \\ ${ }^{\mathrm{d}}$ University of New South Wales, Australia \\ 'University of Sydney, Australia \\ ${ }^{\mathrm{f}}$ University College London, UK
}

\begin{abstract}
We describe recent research into devices based on fibre Bragg gratings in polymer optical fibre. Firstly, we report on the inscription of gratings in a variety of microstructured polymer optical fibre: single mode, few moded and multimoded, as well as fibre doped with trans-4-stilbenmethanol. Secondly, we describe research into an electrically tuneable filter using a metallic coating on a polymer fibre Bragg grating. Finally we present initial results from attempts to produce more complex grating structures in polymer fibre: a Fabry-Perot cavity and a phase-shifted grating.
\end{abstract}

Keywords: Polymer optical fibre, fibre Bragg grating, sensor, filter.

\section{INTRODUCTION}

At the moment there is considerable interest in the use of polymer optical fibre (POF) in a variety of applications. In several countries POF is seen as a good candidate as a digital transmission medium in fibre-to-the-home applications and for home networking. POF is also increasingly being used in automotive data buses, while one of the largest markets, in terms of the sheer length of fibre used, is in illumination applications. All of these markets are possible because POF based systems are seen as being low cost; this applies to both the intrinsic production cost of the fibre itself as well as the cost of its installation, including the making of connections. The communications applications mentioned all involve short transmission distances where the much higher loss of POF in comparison with silica fibre and its high intermodal dispersion are not serious disadvantages. The high intermodal dispersion in these systems arises because of the predominant use of very large core multi-mode fibres, which makes it easy and therefore cheap to couple to low cost, large-area sources and to make fibre-to-fibre connections.

A number of workers have developed techniques for sensing using POF which also follow this low cost paradigm ${ }^{1}$ and some of these devices are beginning to be commercialised. At the same time, there have been some recent technological developments which open up new possibilities for POF based sensors and devices. These are:

- the development of single-mode step index optical fibre, ${ }^{2}$

- the demonstration of the recording of fibre Bragg grating (FBG) filters in POF; ${ }^{3}$

- the production of microstructured or photonic crystal polymer optical fibre (mPOF). ${ }^{4}$

Whilst, as we shall explore in this paper, these technologies offer tremendous scope for the realisation of novel devices, for the foreseeable future they are unlikely to offer very low costs. This is partly due to the underlying cost of production but also often due to the need for sources compatible with single mode fibre, and the difficulty (and hence expense) of connecting single mode polymer fibres. To justify work in this area, one then has to look for other advantages over the very much more mature silica fibre technology. Fortunately it is not difficult to find several major factors that can act in POF's favour for certain applications.

* d.j.webb@aston.ac.uk; phone: +44 (0)121 204 3541; http://www.ee.aston.ac.uk/research/prg/index.html

Optical Sensing II, edited by Brian Culshaw, Anna G. Mignani, Hartmut Bartelt, Leszek R. Jaroszewicz, Proc. of SPIE Vol. 6189, 618901, (2006) · 0277-786X/06/\$15 - doi: 10.1117/12.662306 


\subsection{Yield strain}

Pristine silica optical fibre can survive strains of up to around 6\%. However, the stripping of the fibre coating associated with the inscription of fibre Bragg grating strain sensors tends to reduce the yield stress and it is common to expect a working maximum strain of around $1 \%$. There are a number of applications where the strains encountered can easily exceed this value; for example, many modern composite materials can survive much higher strains. POF has both a much lower Young's modulus and a much higher yield strain than silica fibre. For example, depending on the drawing conditions, PMMA based POF can have an elastic limit of around $10 \%$ and will survive an elongation of around three times its original length before breaking.

\subsection{Temperature sensitivity}

The physical properties of POF (e.g. refractive index or physical length) are generally much more sensitive to temperature than those of silica. For some applications, this can be a disadvantage; for example WDM filters must not drift unduly with temperature. However, in other situations it can be a positive advantage. Fibre Bragg grating temperature sensors in POF will show a much greater wavelength shift for a given temperature change than their silica counterparts. This will either allow the use of lower resolution, simpler and therefore cheaper interrogation systems or alternatively enable the sensors to be used in demanding applications where only small temperature changes are to be expected, for example temperature sensing in vivo.

The higher temperature sensitivity of POF based devices allows device properties to be readily controlled thermally. For example, the pass band of a Bragg grating based filter could be easily tuned over several nanometres with a relatively modest temperature change.

\subsection{Material}

Due to its organic nature, the chemical properties of polymer fibre are of course very different to those of the inorganic silica. Advantage could be taken of this fact by making use of the huge range of organic chemistry techniques to modify the fibre structure; this could be done to enhance the non-linear optical properties, to provide amplification or to sensitise the fibre to a particular chemical or biochemical species. Organic dopants can never be used with silica fibre since they would be inevitably destroyed during the high temperature fibre drawing process, whereas the much more modest temperature used with POF (around $200{ }^{\circ} \mathrm{C}$ ) will normally not denature the material.

\subsection{Biocompatibility}

We have already alluded to the use of plastic optical fibre Bragg gratings as in vivo sensors. For this and other medical applications, POF is inherently more biocompatible than silica. Whilst clinicians have for many years been at ease with introducing various polymer devices into the body for extended periods, the necessity of guarding against a fibre breakage could considerably complicate the design of any silica fibre probe.

\section{FIBRE BRAGG GRATINGS IN POF}

Fibre Bragg gratings consist of an axially periodic variation in the refractive index of the core of an optical fibre ${ }^{5}$ Such a grating acts so as to reflect light in a narrow band of wavelengths around the Bragg wavelength, $\lambda_{B}$, whilst transmitting other wavelengths. The Bragg wavelength is related to the period of the index modulation, $\Lambda$ by

$$
\lambda_{B}=2 n \Lambda,
$$

where, $\mathrm{n}$ is the effective index of the core mode. In silica fibre the gratings are almost invariably produced by illuminating the fibre core with an axially periodically varying intensity pattern of UV light, produced either by interfering two coherent beams, or else using a phase mask, making use of the natural photosensitivity of the fibre, or enhancing the photosensitivity with hydrogen loading 5 . Such gratings in silica fibre have been intensively researched worldwide for more than 15 years, as they find many applications in wavelength division multiplexing, dispersion compensation and as temperature and strain sensors 5 . POF has been known to be photosensitive since the early seventies ${ }^{6}$, but it was only with the development of single mode POF that it became interesting to try to develop FBG 
technology in $\mathrm{POF}^{7}$. The first POF Bragg gratings were reported in 1999 by researchers at the University of New South Wales in Australia ${ }^{3}$. The gratings were produced in step-index single mode PMMA based fibre.

\section{FIBRE BRAGG GRATINGS IN MPOF}

More recently, considerable progress has been made in the development of microstructured polymer optical fibre, where guiding of the core mode arises as a result of the presence of a surrounding array of air holes in a fibre formed from a single material (PMMA in the work reported here). The use of a microstructured fibre brings a number of potential advantages to device fabrication.

- The dispersion of the fibre depends on the particular geometry used, thus there is the potential for tailoring the dispersion properties in a relatively straightforward way. As an example, such fibre can display endlessly single mode behaviour. Furthermore, the ability to tailor dispersion might be particularly important where long period gratings are concerned, as with these devices the sensitivity to a range of measurands depends strongly on differential effects between the dispersions of the core and cladding 8 .

- At the preform stage, the holes (typically produced using drilling) offer a convenient means of introducing dopants into the core region, which can enhance the optical properties in various ways.

- In the fibre, the holes offer a means of getting gas or liquid in contact with the electric field of the light in the core, enabling chemical sensing to take place over long path lengths but with very small volumes of analyte.

The microstructured geometry offers a particular challenge in terms of the grating inscription. For FBG production in step-index fibres it is usual to focus the writing beam down into the fibre core; however, the presence of several rings of holes surrounding the core in the microstructured fibre will tend to scatter the incident beam, which might be expected to lead to a significant reduction in the optical intensity in the core region. Recent success in fabricating FBGs in microstructured silica fibre with a small germanium doped $\operatorname{core}^{9}$ and in pure silica microstructured fibre ${ }^{10}$ using a pulsed $193 \mathrm{~nm}$ laser, as well as inscription in undoped silica microstructured fibre using a $267 \mathrm{~nm}$ femtosecond lase ${ }^{11}$, have provided confidence that this effect did not necessarily preclude grating inscription, but in the aforementioned cases high energy pulses were always deemed necessary to compensate for the reduction in the optical intensity caused by hole-induced scattering.

\subsection{Fibre production}

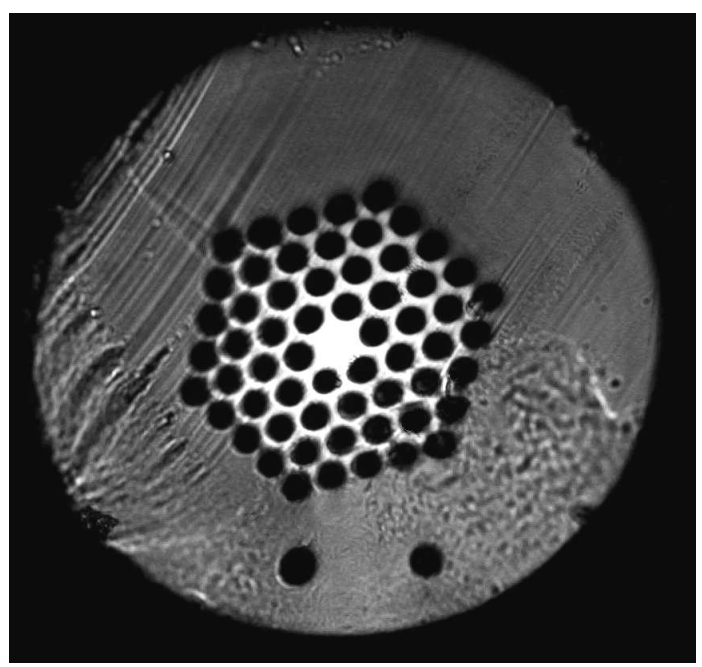

Figure 1: Optical microscope image of the cross-section of the mPOF.

The fibre used in this work was made from a PMMA primary preform of $70 \mathrm{~mm}$ diameter, into which the desired pattern of holes was drilled. The primary preform was drawn to a diameter of $12 \mathrm{~mm}$ to form the secondary preform, 
and the secondary preform was drawn to fibre directly. In mPOF fabrication the secondary preform is usually sleeved to increase its outer diameter. This step was omitted in this case, to minimise the material surrounding the structure and hence to minimise UV absorption outside the core region. The few-moded mPOF had an outside diameter of $160 \mu \mathrm{m}$ and a core diameter of $13 \mu \mathrm{m}$; the core being surrounded by 60 air holes with diameters of $5.5 \mu \mathrm{m}$ and a separation of $10 \mu \mathrm{m}$; see figure 1 .

\subsection{Inscription}

The ends of the mPOF were prepared for light transmission initially by cutting the fibre using a cold sharp razor blade to create a flat end face (for later work a heated blade has provided better results ${ }^{12}$ ). An $11.4 \mathrm{~cm}$ length of mPOF was then mounted in three v-grooves which were attached to two $\mathrm{x}, \mathrm{y}, \mathrm{z}$ translation stages. FBG inscription was undertaken using the standard phase mask technique utilising a mask with a pitch of $1060.85 \mathrm{~nm}$ designed to produce a FBG in silica at around $1536 \mathrm{~nm}$. A continuous wave helium cadmium laser with an output wavelength of $325 \mathrm{~nm}$ and a power of $30 \mathrm{~mW}$ was used to inscribe the FBG. Two plano-convex cylindrical lenses with $10 \mathrm{~cm}$ focal lengths were incorporated in the system, one in the usual position before the phase mask, which served to focus the light down towards the core, and the other at a distance of $56.5 \mathrm{~cm}$ from the mPOF. The second lens was used to expand the $1.8 \mathrm{~mm}$ diameter laser beam to cover approximately $1 \mathrm{~cm}$ of the mPOF. Figure 2 shows the experimental arrangement.

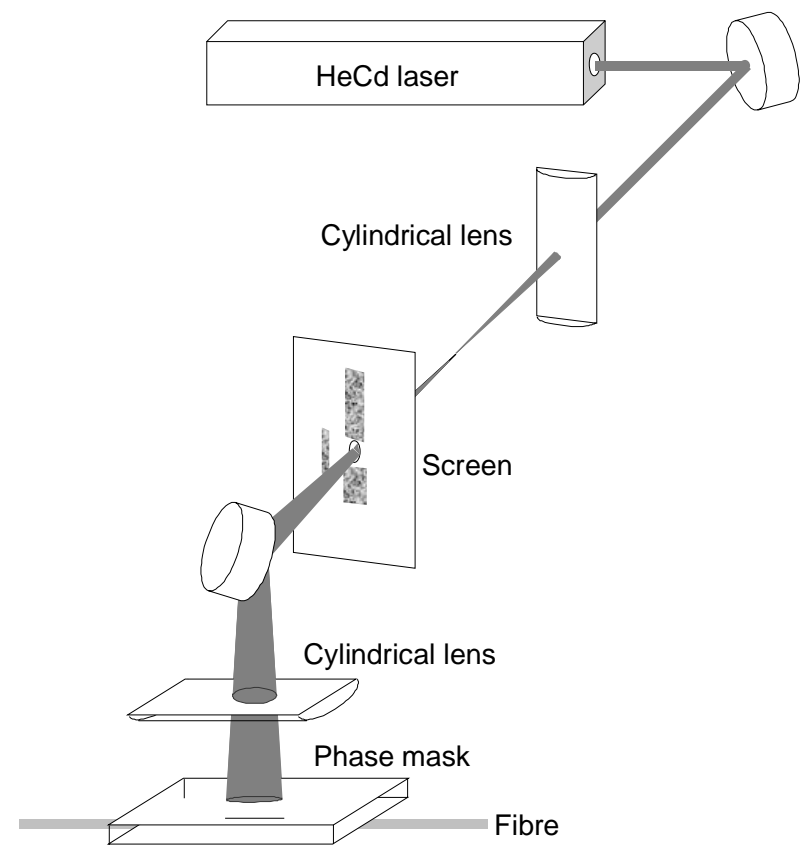

Figure 2: Experimental arrangement.

The orientation of the fibre geometry with respect to the incident laser beam is critical for FBG fabrication in mPOF. Whereas in step-index fibre the alignment affects the quality of the grating, incorrect alignment of the mPOF during FBG inscription can prevent grating inscription; in particular it is essential that the fibre is correctly oriented rotationally about its axis with respect to the incident beam. Inspection of the back reflections of the HeCd beam from the mPOF revealed two different diffraction patterns as the fibre was rotated; one thin and bright and the other broad and dull. It was found to be essential that the back reflection was thin and bright for inscription. Examples of the two patterns can be seen in figure 3 .

Having aligned the mPOF with the incident beam, light was then coupled into the fibre so that grating growth could be monitored. A SMF $2 \times 2$ coupler designed to operate in the telecommunications C-band was used so that the grating could be monitored in reflection. One of the output arms of the coupler was tied off to eliminate back reflections from that arm, whilst the other output arm had an AFC/PC pigtail spliced on to it. The AFC/PC connector was butted up 
against the launch end of the mPOF with a small amount of index matching gel to reduce the Fresnel reflections from the end and aid coupling into the mPOF. A SMF patchcord was used to monitor the transmission through the mPOF on an OSA. Alignment was carried out by connecting a FC/PC pigtail spliced on to an input arm of the coupler to a HeNe laser with an output of $15 \mathrm{~mW}$ at a wavelength of $633 \mathrm{~nm}$. The translation stage was then adjusted to maximise throughput of the $633 \mathrm{~nm}$ light. The HeNe laser was then replaced with an ASE light source (Thorlabs ASE-FL7002-C4, $5 \mathrm{~mW}$ output power) with an operating wavelength range of $1530-1610 \mathrm{~nm}$. The launching alignment was altered fractionally to maximise the throughput for these wavelengths. The OSA was then swapped to the second input arm of the coupler to monitor the reflection from the mPOF.
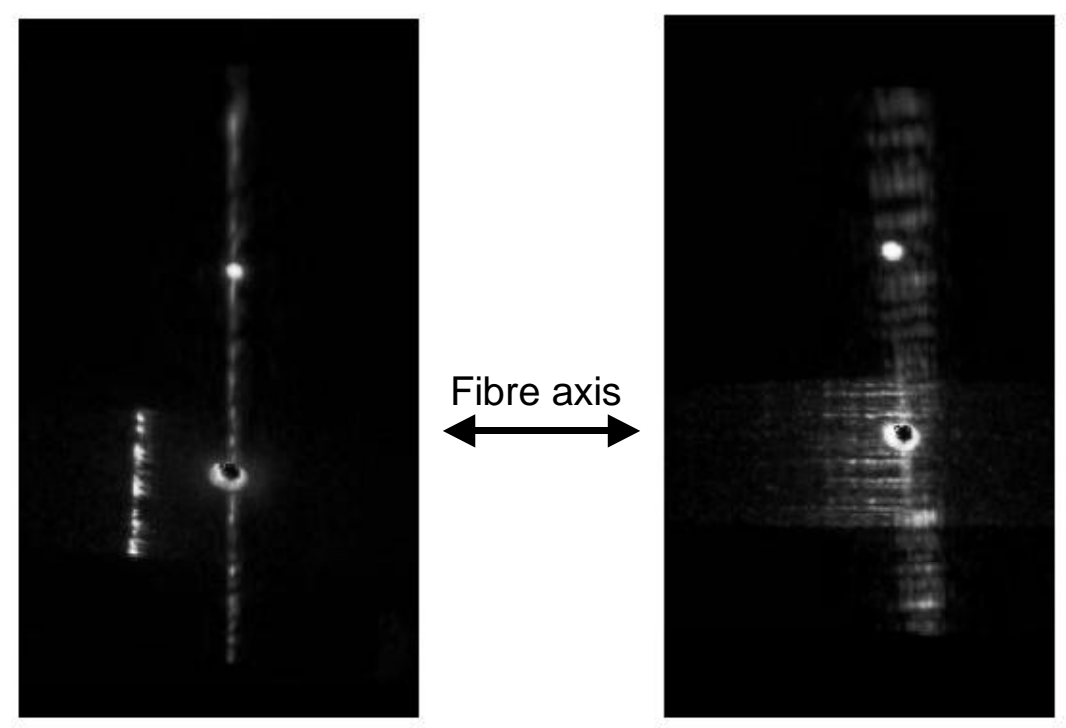

Figure 3: Backscattered diffraction patterns of HeCd laser viewed by fluorescence; the pattern on the left was needed for successful inscription.

Exposure lasted for 60 minutes at a room temperature of $29^{\circ} \mathrm{C}$ before saturation of the grating occurred. The resulting FBG had a Bragg wavelength of $1570 \mathrm{~nm}$, a width (FWHM) of a little under $1 \mathrm{~nm}$, a length of $1 \mathrm{~cm}$ and a reflective power above the noise level of $7 \mathrm{~dB}$. Figure 4 shows the grating profile (OSA bandwidth $=0.2 \mathrm{~nm}$ ) and the growth characteristics. Evidence for the few-moded structure of the reflectivity profile can be clearly seen.
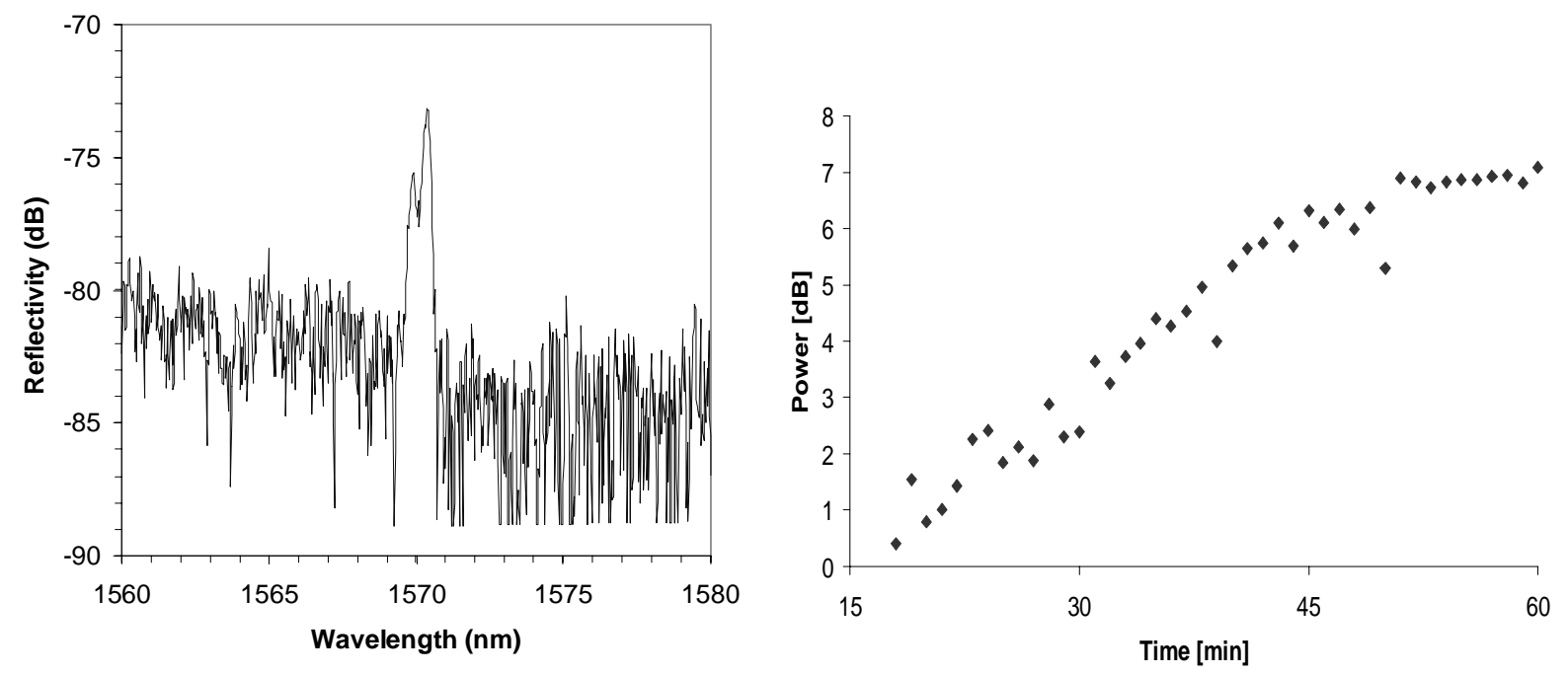

Figure 4: Left: reflection profile of fabricated FBG; Right: growth of signal to noise ratio. 
In the absence of index matching gel, the returned signal was observed to be roughly comparable to the Fresnel reflection from the end of the fibre launching light into the POF. The attenuation of the POF at the grating wavelength is around $72 \mathrm{~dB} / \mathrm{m}$; consequently, the $3 \mathrm{~cm}$ distance to the grating from the input face of the POF implies that the reflectivity of the grating will be at least $10 \%$. The actual value could be higher since this calculation fails to take into account losses in coupling from the silica fibre to the POF.
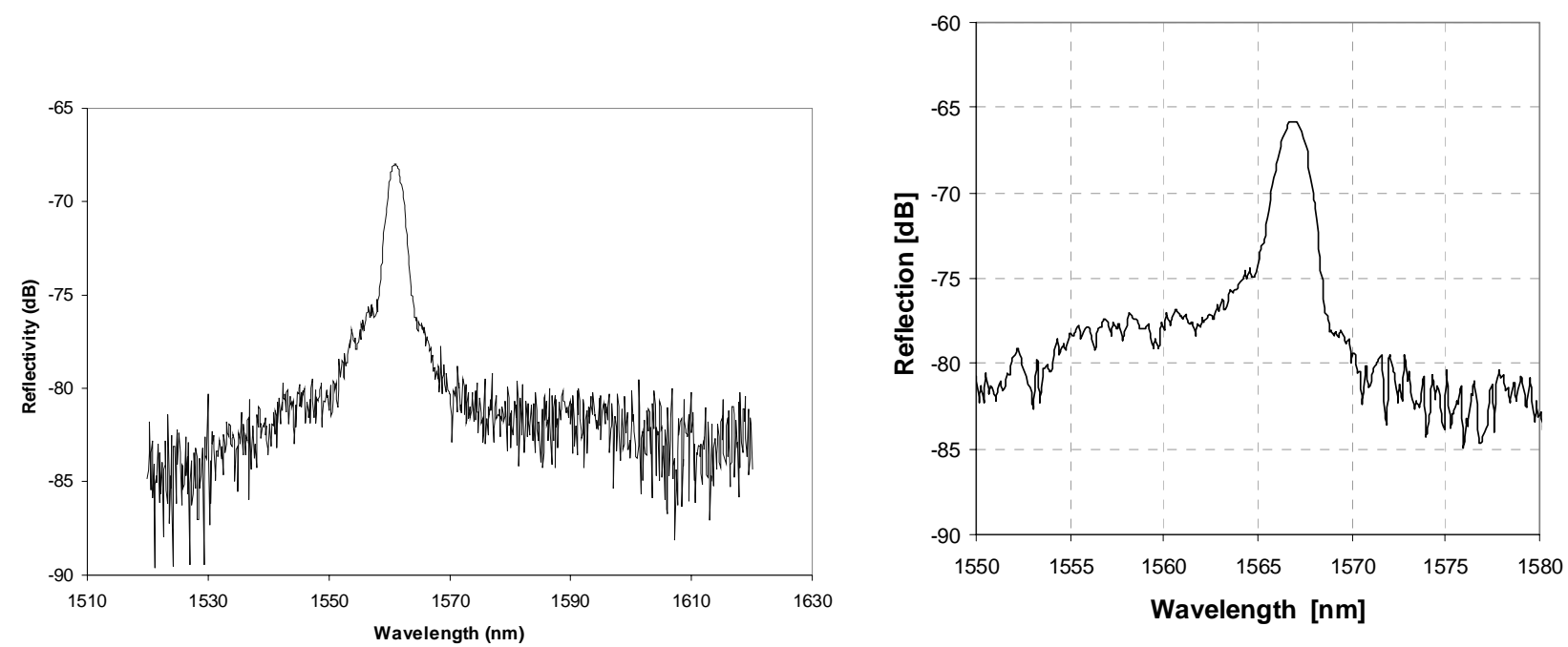

Figure 5: Reflection spectra from grating in single mode POF (left) and multimode fibre (right)

Following this first success, we have been able to write Bragg gratings in several other types of mPOF. Figure 5 shows reflection spectra obtained with endlessly single mode and $50 \mu \mathrm{m}$ core multimode mPOF. The encouraging thing about the multimode fibre result is that the grating bandwidth still has the relatively narrow bandwidth of less than $3 \mathrm{~nm}$. Given the enhanced temperature sensitivity of POF compared to silica fibre, in a thermometric application it should be possible track such a peak with a similar or better resolution to a typical FBG in silica fibre, while the larger core diameter opens up the possibility of making use of cheaper, broad area emitters.

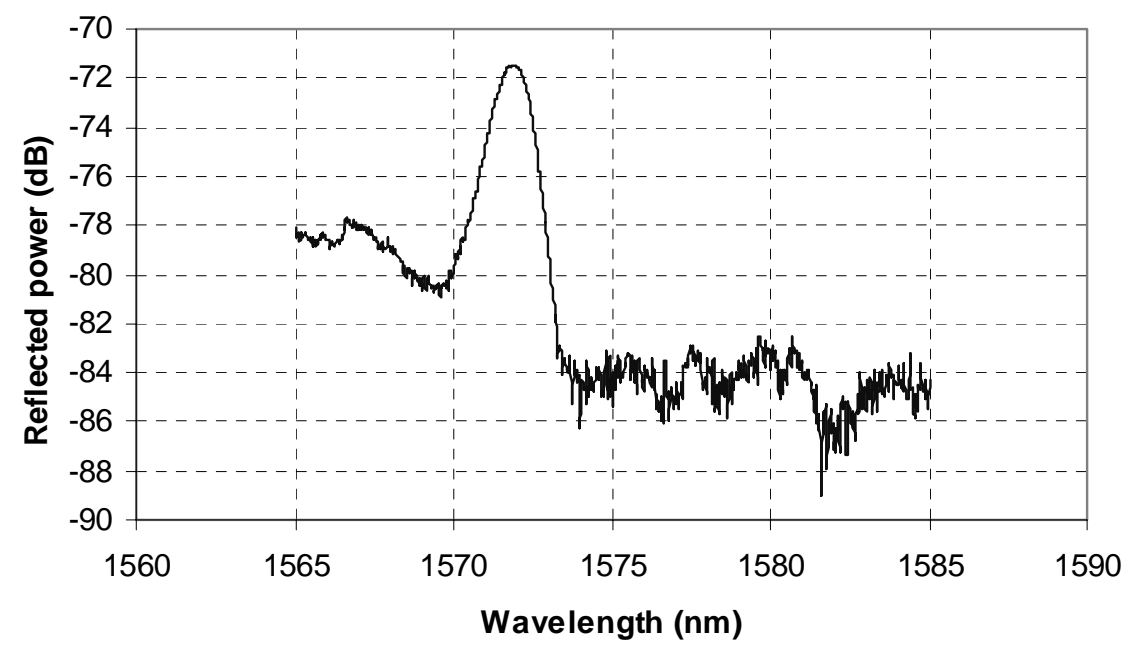

Figure 6: Reflection spectrum for a grating recorded in trans-4-stilbenmethanol doped PMMA mPOF. 
Figure 6 shows the first results from a single mode mPOF doped with trans-4-stilbenmethanol. With the nominally pure PMMA fibre used in our experiments, it typically takes $15-20$ minutes for a grating to begin appearing (see figure 3 for example). In the case of the doped fibre, the grating reveals itself after only 8 minutes. Work continues aimed at improving the grating reflectivity and reducing the inscription time still further.

\section{ELECTRICALLY TUNEABLE GRATINGS IN POF}

POFBGs have already been shown to have a high temperature sensitivity ${ }^{13}$, and hence be suitable candidates for producing a widely tuneable filter by heating the fibre. Using the system described above, FBGs were inscribed into step-index few moded POF, after which the region around the grating was coated with a palladium/copper metallic layer. Metal deposition was initiated by the use of a vacuum UV (VUV) light source operating at $172 \mathrm{~nm}$ and at low (room) temperature, see figure 7. At this wavelength the penetration depth of the light source is on the order of a micron and therefore there is no damage to the grating device, but the surface of the sample undergoes a chemical roughening, as the light initiates bond scission. The VUV exposure time ranged from 1 to 10 minutes. The dangling polymer bonds can then bond both physically and chemically to metallic particles. This is of great advantage as there are very few approaches for coating polymers with metal at room temperature and much higher temperatures would have an impact on the performance and spectral characteristics of the grating. Furthermore, the coating took place in a low vacuum of $10^{-2} \mathrm{mbar}$, which again is not enough to affect the basic fibre and grating properties. This approach of decomposing metal organic films with incoherent VUV excimer lamps at low temperature is ideal for the manufacture of thin film microcircuits and devices, proving to be an efficient technique for the preparation of uniform thin films. The film thickness is controlled by variation of the reaction parameters - the concentration of precursor and the UV-irradiation time.

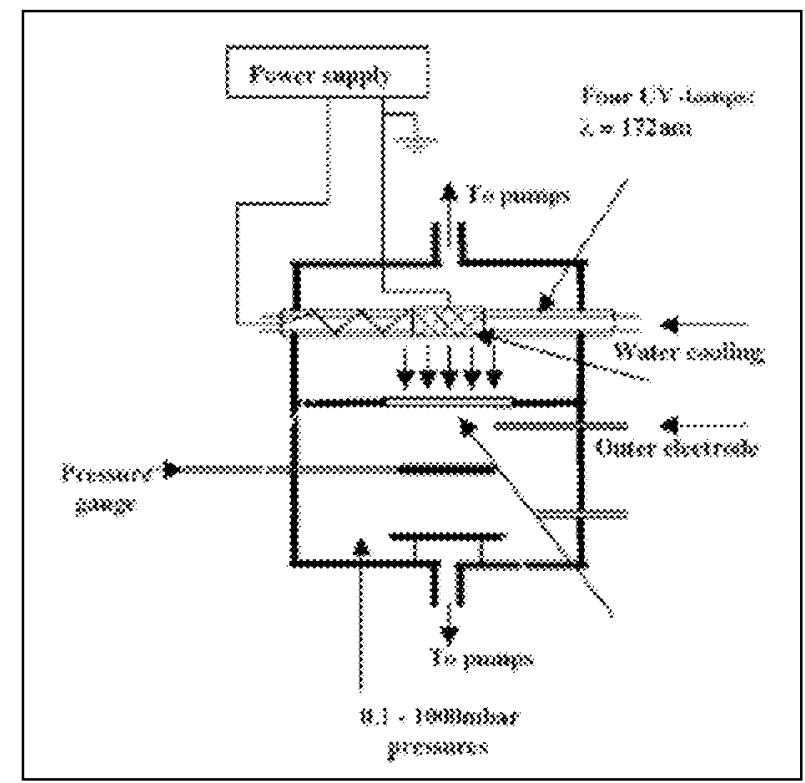

Figure 7: Schematic diagram of the coating procedure.

\subsection{Modelling}

The response of a thin film heated Bragg grating for a glass optical fibre has been characterised, but this is the first such demonstration for a polymer-based fibre Bragg grating. Here we anticipate some differences between the thermal properties of the glass and polymer based devices, principally the thermal diffusion and radiation dissipation, given the different thermal conductivities and emissivities of glass and polymer materials.

In order to study the behaviour of the polymer-based tuneable fibre Bragg grating we consider a differential equation that describes the temperature distribution and thermal flow in the fibre. We make the assumption that the coating is 
sufficiently uniform and greater in length than the grating to give a uniform temperature distribution across the grating length, so only the radial distribution and thermal flow along the grating length are of importance. The device can therefore be essentially modelled as a polymer cylinder with a thin-film thermal source on the surface enclosed by air. In this way the heat diffuses into the fibre core and the surrounding air, in addition to radiation. Considering energy conservation within the fibre,

$$
\frac{\partial T(x, t)}{\partial t}=\kappa \frac{\partial^{2} T(x, t)}{\partial x^{2}}+\frac{P_{\text {in }}(x, t)-P_{\text {out }}(x, t)}{\rho c_{p} \pi R_{\text {fibre }}^{2}}
$$

where $x$ is the distance along the fibre, $T(x, t)$ is the fibre temperature, $\kappa, \rho$ and $c_{p}$ are the thermal diffusivity, density and constant pressure heat capacity of the polymer (PMMA), $R_{\text {fibre }}$ is the fibre radius. $P_{i n}(x, t)$ is the heating generated by the thin film heater, and $P_{\text {out }}(x, t)$ is the rate of heat loss from the fibre to the air. In general $P_{\text {in }}(x, t)=I^{2}(t) R(x)$, where $R(x)$ is the local resistance per unit length of the coating and $I(t)$ is the electrical current. Furthermore, if we assume that the rate of heat loss is proportional to the change in temperature then $P_{\text {out }}(x, t)=a\left|T(x, t)-T_{\text {air }}\right|$, where $a$ is a parameter that may be temperature dependent and represents a lumped heat transfer coefficient due to heat transfer from the surface of the device. The heat loss from the metal to the air follows radiative heat transfer from two grey bodies of the form

$$
\propto 2 \pi R_{\text {fibre }} \sigma\left(T(x . t)^{4}-T_{\text {air }}^{4}\right)
$$

where $\sigma$ is the coefficient of thermal radiation dissipation. However, given the low temperature range one can approximate this to $\propto\left(T(x . t)-T_{\text {air }}\right)$. Given that the rate of heat loss is linearly proportional to the change in temperature we can write,

$$
P_{\text {out }}(x, t)=2 \pi R_{\text {fibre }} h T(x, t)
$$

where $\mathrm{h}$ is the surface conductance of the fibre, which characterises the heat flow out of the fibre. One can introduce the constant $a$ as follows,

$$
\frac{P_{\text {out }}(x, t)}{\rho c_{p} \pi R_{\text {fibre }}^{2}}=a T(x, t)
$$

hence $a$ is related to $h$ by

$$
a=\frac{2 h}{\rho c_{p} R_{\text {fibre }}}
$$

In addition to the above terms there are radial heat flow terms that may have to be considered,

$$
2 \pi R_{\text {fibre }} \kappa \frac{\partial T}{\partial r}_{r=R f i b r e} \text { and } 2 \pi R_{\text {fibre }} \kappa_{\text {air }}{\frac{\partial T_{\text {air }}}{\partial r}}_{r=R f i b r e}
$$

specifically the difference

$$
2 \pi R_{\text {fibre }}\left[\kappa \frac{\partial T}{\partial r}_{r=\text { Rfibre }}-\kappa_{\text {air }}{\frac{\partial T_{\text {air }}}{\partial r}}_{r=\text { Rfibre }}\right]
$$


where $\kappa_{a i r}$ and $T_{a i r}$ are the thermal diffusivity of air and the air temperature, respectively. This term is important as it accounts for temperature gradients that may exist radially across the grating. Given that the thermal conductivity of PMMA $(0.17 \mathrm{~W} / \mathrm{mK})$ is only 7 times that of air $(0.025 \mathrm{~W} / \mathrm{mK})$, compared with 55 times for silica glass $(1.38 \mathrm{~W} / \mathrm{mK})$ one should consider the validity of whether one can ignore radial gradients and consider only heat flow along the length of the fibre. The heat flow through and along the surface of the fibre may be characterised by the Biot number, $B i=h$ $R_{f i b r e} / k$, where $h$ is the surface conductance of the fibre. When the Biot number is sufficiently small, meaning that the thermal conductance over the length $R_{f i b r e}$ is far greater than the surface conductance then radial thermal gradients become negligible, this translates to a Biot number $B i<0.1$. Hence it is clear that a measurement of $a$ will lead to an estimate of the surface conductance and the Biot number of the fibre, from which we can determine whether the fibre can be treated as a one-dimensional system. $a$ constitutes a system time constant, therefore we should make measurements of the filter's wavelength shift as a function of time in response to a sudden current input.

\subsection{Wavelength shift of the filter}

In order to characterise the tuneable filter an electrical connection was made to the metal-coated polymer by using silver loaded paint and fine copper wires. The passage of current through the film, via the copper wires, resulted in joule heating that increased the temperature of the fibre. The copper wires were placed with a separation of $12 \mathrm{~mm}$ encompassing the grating that had a length of less than $1 \mathrm{~cm}$. For this type of device cooling is passive in nature and occurs through convective and conductive flow of heat to the surrounding air. The spectral reflectivity, and its changes in response to current flowing through the thin copper film, was measured using a broadband light source and optical spectrum analyser. The total resistance of the film encompassing the grating, and the connection via the copper wires and silver-loaded paint, was measured to be $3.2 \Omega$.

Figure 8 shows the wavelength shift induced by joule heating, on passing a direct current through the metallic film coating the polymer fibre. We note that throughout the wavelength tuning the grating spectrum remained unchanged, and this observation is consistent with a spatially uniform heating of the grating. It is noted that the wavelength to input power coefficient is $-13.4 \mathrm{pm} / \mathrm{mW}$. Figure 9 shows the spectrum of the grating for several values of input power.

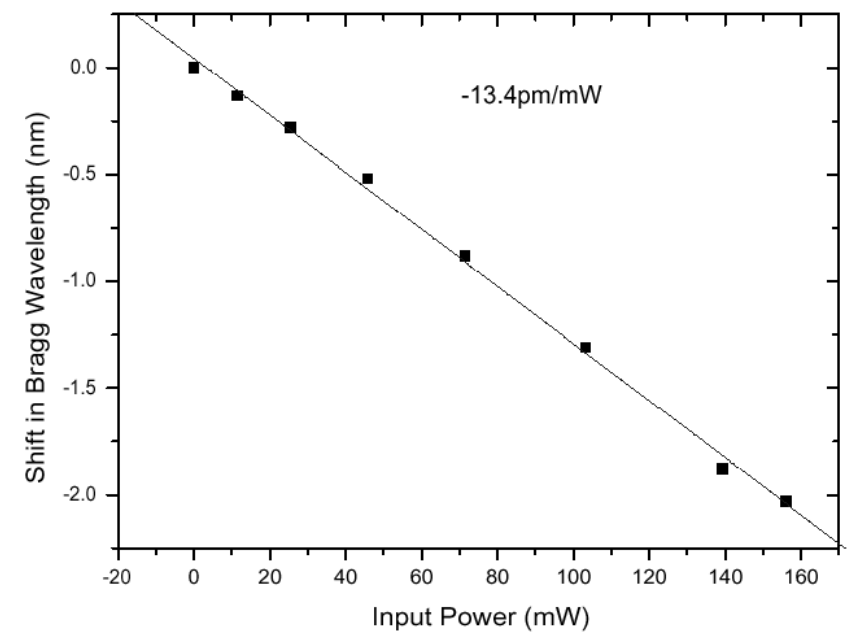

Figure 8: The wavelength shift of the Bragg grating as a function of input power. 


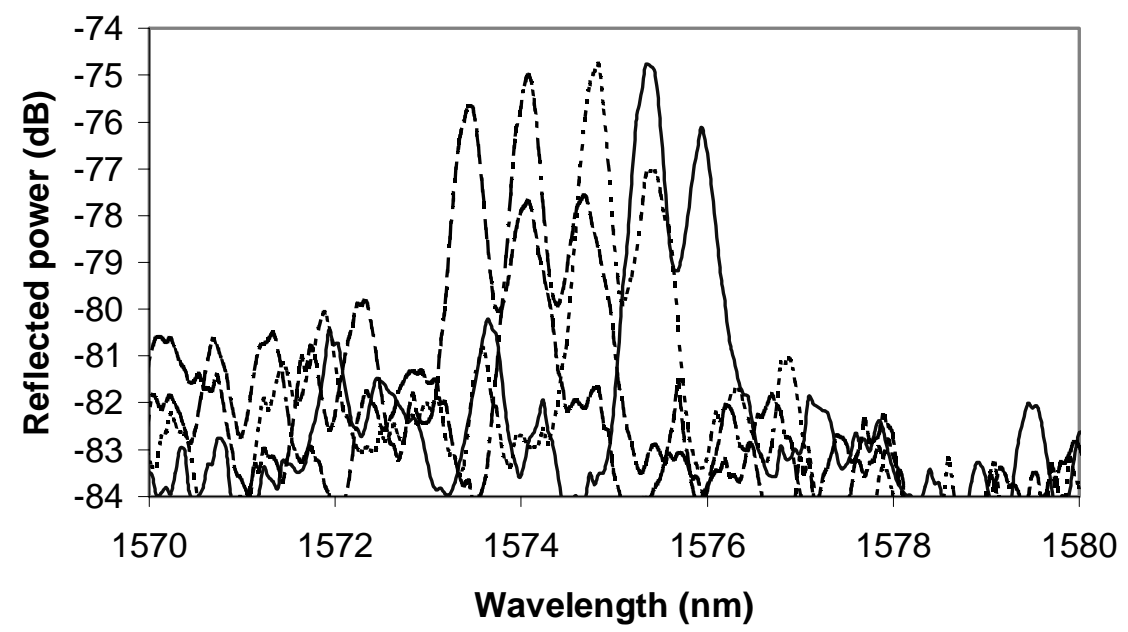

Figure 9: Wavelength spectrum showing four typical traces for powers varying from $150,100,50,0 \mathrm{~mW}$, respectively from left to right.

\subsection{Response time of filter}

When the input power is uniform the temperature variation as a function of time is

$$
T(t)=\frac{P_{i n}^{\prime}}{a}[1-\exp (-a t)]
$$

where $\mathrm{P}_{\text {in }}$ ' is related to the input power, but depends on the thermal capacity of the PMMA and the metal film. As noted earlier, a measurement of $a$ yields information regarding the rate of heat flow out of the fibre. Figure 10 shows the rise time of a thermally tuned fibre Bragg grating, with data collected for three different heating powers applied suddenly at $\mathrm{t}=0 \mathrm{~s}, 45.8,71.5$, and $103.2 \mathrm{~mW}$. The curves correspond to fits that use a single exponential form, from which we have determined the time constant, $a=1.7 \mathrm{~s}^{-1}$. There is no apparent or systematic dependence on the applied power.

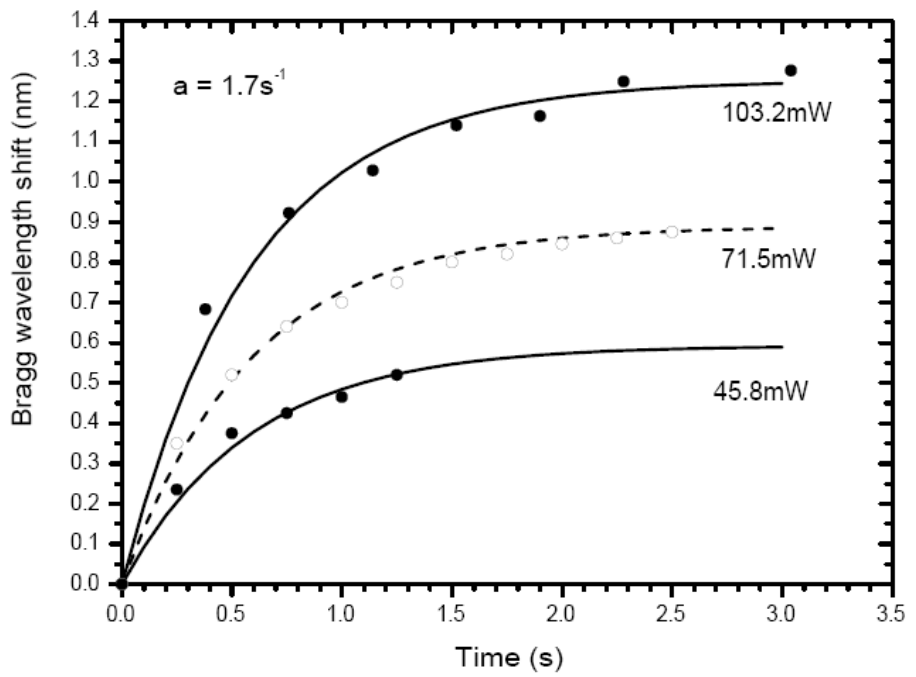

Figure 10: Rise time of a thermally tuned fibre Bragg grating, with data collected for three different heating powers applied suddenly at $\mathrm{t}=0 \mathrm{~s}: 45.8,71.5$, and $103.2 \mathrm{~mW}$. The time constant $a=1.7 \mathrm{~s}^{-1}$. 
Having determined $a$ we can now evaluate the surface conductance $h$ and the Biot number. Using the geometric and material properties of the fibre $\left(\rho=1190 \mathrm{~kg} / \mathrm{m} 3, c_{\mathrm{p}}=1450 \mathrm{~J} / \mathrm{kgK}, R_{\text {fibre }}=75 \mu \mathrm{m}, \kappa=0.17 \mathrm{~W} / \mathrm{mK}\right), h$ is found to be $110 \mathrm{~W} / \mathrm{m}^{2} \mathrm{~K}$, and $\mathrm{Bi}$ is 0.049 . We note that $\mathrm{Bi}$ is less than 0.1 , and this is compatible with a treatment of the fibre as a simple one dimensional system.

\section{GRATING BASED INTERFEROMETRIC DEVICES}

Amongst the range of fibre sensor devices, fibre interferometers provide some of the highest measurement resolutions. It is possible to construct fibre interferometers by using directional couplers to carry out amplitude division in a Michelson or Mach-Zehnder configuration, or to use the end reflections from a section of fibre to define a Fabry-Perot or Fizeau cavity. Such devices can, however, possess a number of disadvantages, such as their bulk or the difficulty of incorporating them into systems. Fabry-Perot cavities can be much more conveniently produced in silica fibre using FBGs as the mirrors; not only does this allow for a very compact sensor, but it also allows the possibility of wavelength division multiplexing of multiple sensors.

We have been carrying out preliminary research aimed at establishing the feasibility of producing such devices in POF. Figure 11 shows reflection spectra from what we believe are the first FBG cavities written in PMMA based step-index POF. The left-hand spectrum is produced by a Fabry-Perot cavity formed from two $1 \mathrm{~cm}$ long gratings separated by about $3 \mathrm{~cm}$. The approximately sinusoidal nature of the fringes superimposed on the grating reflection profile is indicative of a low finesse cavity, caused partially by the large attenuation in this wavelength range. In fact for many applications, low finesse cavities are preferable.

An alternative means of producing an interferometric cavity is by means of a phase shifted grating. A phase shifted grating is a uniform fibre Bragg grating which has a phase shift in the centre of the grating planes. The phase change effectively creates two gratings out of phase with each other which form a wavelength selective Fabry-Perot resonance allowing light at the resonance to penetrate the stop-band of the original grating. The phase shift can be introduced by several different methods:

- Using a phase mask with a shift incorporated in to the design;

- Post-processing of a grating by exposing a region of the grating to further UV radiation;

- Post-fabrication processing using localised heat treatment.

The second method was utilised to create a phase shifted FBG in POF. A $1 \mathrm{~cm}$ standard uniform grating was fabricated to saturation. The unexpanded laser beam, approximately $1 \mathrm{~mm}$, was then used to blank beam the centre of the grating with UV. The resulting phase shifted grating can be seen in the right hand spectrum of figure 10 . The high attenuation is again partially responsible for a lack of sharpness in the central reflection notch; a move to gratings designed for visible wavelengths should result in a much sharper spectral feature, the wavelength of which could be monitored with correspondingly higher precision.

\section{CONCLUSIONS}

For the reasons listed earlier, we believe that polymer optical fibre devices have the potential to be exploited in a range of applications. In this paper we have described some of our work towards realising that potential. It must be recognised though that these are early days and there is some way to go before this technology can be considered to be anything like as mature as its silica counterpart. There are several areas where progress is required: the strength of gratings in mPOF needs to be improved, either by optimising the inscription arrangement or by further exploring the use of doped fibres; a move to shorter Bragg wavelengths will allow advantage to be taken of the much lower POF attenuation available in the visible spectrum; a reliable technique for connecting single mode POF into a silica fibre system would prove very useful. We are confident that these issues can be satisfactorily resolved. 

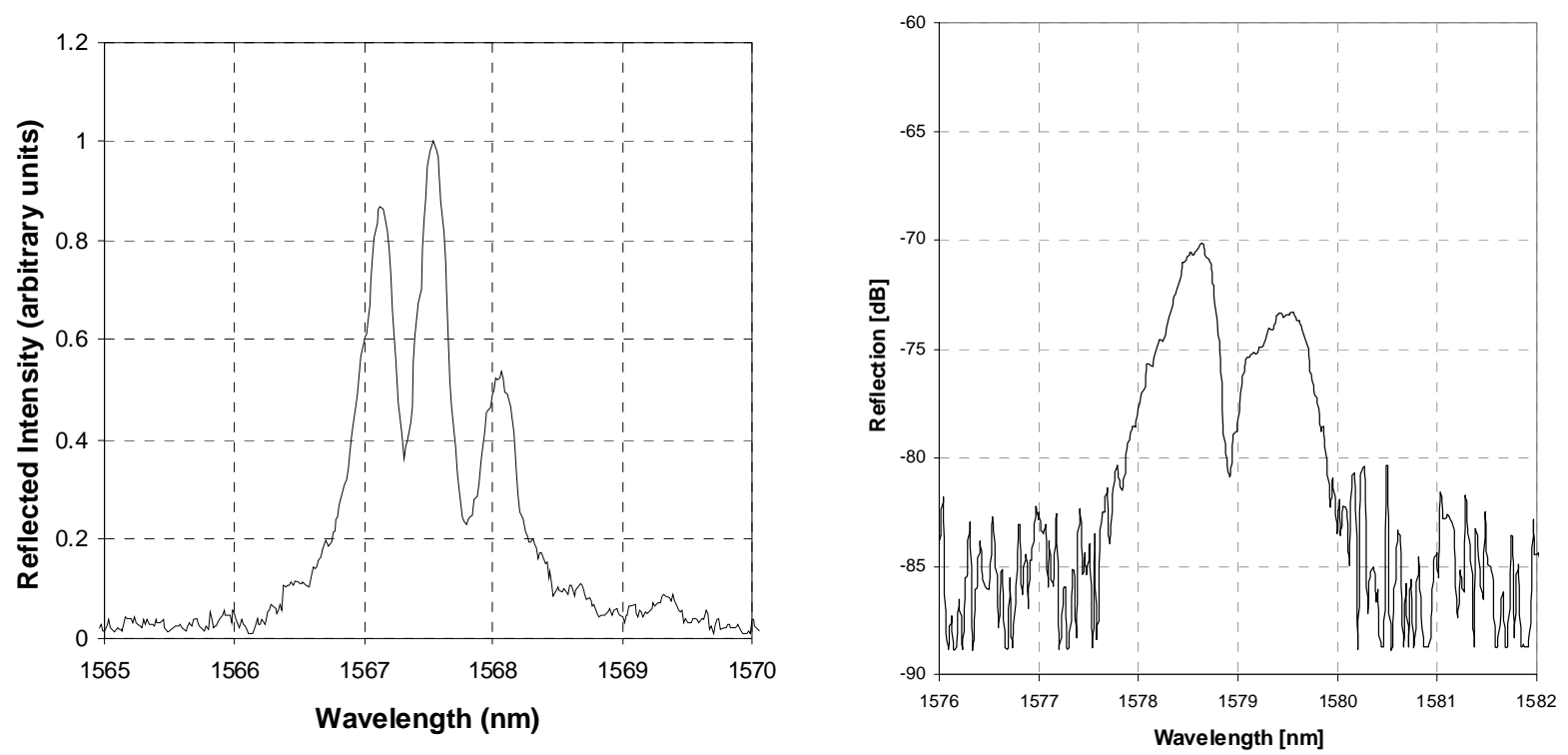

Figure 11: Reflection spectra from: left - Fabry-Perot cavity; right $-\pi$-shifted grating.

\section{ACKNOWLEDGEMENTS}

The authors would like to thank the following sources of financial support: UK Engineering and Physical Sciences Research Council; European Union Eureka project POLYFILTRO.

\section{REFERENCES}

${ }^{1}$ RJ Bartlett, R Philip-Chandy, P Eldridge, DF Merchant, R Morgan and P Scully, "Plastic optical fibre sensors and devices," Transactions of the Institute of Measurement and Control, 22, 5, pp431-457, 2000

${ }^{2}$ D. Bosc and C. Toinen, "Fully polymer single-mode optical fiber", IEEE Photo. Tech. Lett. 4(7), 749-751, 1992

${ }^{3}$ Z. Xiong, G. D. Peng, B. Wu and P. L. Chu, "Highly tunable single-mode polymer optical fibre grating", IEEE Photonic Technology Letters, vol.11, no.3, 352-354, 1999

${ }^{4}$ G Barton, MA van Eijkelenborg, G Henry, MCJ Large, J Zagari, "Fabrication of microstructured polymer optical fibres," Optical Fiber Technology, 10, pp. 3250335, 2004

${ }^{5}$ A. Othonos and K. Kalli Fiber Bragg gratings: fundamentals and applications in telecommunications and sensing (Artech House, 1999)

${ }^{6}$ IP Kaminow, HP Weber and EA Chandross, Applied Physics Letters, 18, 497, (1971)

${ }^{7}$ G. D. Peng, "Prospects of POF and Grating for Sensing", Proc of the 15th International Conference of Optical Fiber Sensors, Portland, OR, USA, pp.71-4, 6-10 May, 2002

${ }^{8} \mathrm{~V}$ Bhatia, "Applications of long-period gratings to single and multi-parameter sensing", Opt. Express., vol. 4, no. 11, pp.457-466, 1999

${ }^{9}$ B. J. Eggleton, P. S. Westbrook, R. S. Windeler, S. Spälter, and T. A. Strasser, "Grating resonances in air-silica microstructured optical fibers," Optics Letters, 24, 21, 1460-1462, 1999

${ }^{10}$ N. Groothoff, J. Canning, E. Buckley, K. Lyttikainen and J. Zagari, "Bragg grating in air-silica structured fibers," Optics Letters, 28, 4, 233-235, 2003

${ }^{11}$ L.B. Fu, G.D. Marshall, J.A. Bolger, P. Steinvurzel, E.C. Magi, M.J. Withford and B.J. Eggleton, "Femtosecond laser writing Bragg gratings in pure silica photonic crystal fibres. Electronics Letters, 41, 11, 638-639, 2005

${ }^{12}$ S.H. Law, J.D. Harvey, R.J. Kruhlak, M. Song, E. Wu, G.W. Barton, M.A. van Eijkelenborg, M.C.J. Large, "Cleaving of microstructured polymer optical fibres" Optics Communications, 258, 2, pp. 193-202, 2006

${ }^{13}$ H. Y. Liu, G. D. Peng and P. L. Chu, "Thermal tunability of polymer optical fibre Bragg gratings", IEEE Photonic Technology Letters, Vol.13, No.8, pp.824-826, 2001 